\title{
A spherically symmetric model of anisotropic fluid for strange quark spheres
}

\author{
Abdelghani Errehymy ${ }^{1, \mathrm{a}}$, Mohammed Daoud ${ }^{2,3, \mathrm{~b}}$, El Hassan Sayouty ${ }^{1, \mathrm{c}}$ \\ ${ }^{1}$ Laboratory of High Energy Physics and Condensed Matter (LPHEMaC), Department of Physics, Faculty of Sciences Aïn Chock, University of \\ Hassan II, B.P. 5366, Mâarif, Casablanca 20100, Morocco \\ 2 Department of Physics, Faculty of Sciences, University of Ibn Tofail, B.P. 133, Kenitra 14000, Morocco \\ ${ }^{3}$ Abdus Salam International Centre for Theoretical Physics, Miramare, Trieste, Italy
}

Received: 23 December 2018 / Accepted: 7 April 2019 / Published online: 17 April 2019

(C) The Author(s) 2019

\begin{abstract}
In the present work, we try to find a solution without singularity of Einstein's field equations for the spherically symmetric perfect fluid objects, accurately strange quark spheres, taking into consideration Schwarzschild metric as the outside space-time. An ensemble of inside solutions found on the basis of the simplest linear state equation in the specific form $p_{r}=\alpha \rho-\beta$. The energy density $\rho(r)$, the radial pressure $p_{r}(r)$ and the tangential pressure $p_{t}(r)$ are devoid of any singularity and exhibit a wellbehaved nature within the generalized anisotropic solution for compact spherical object. The generalized TOV equation is very much preserved inside the system and all energy conditions are excellent. The stability of the matter distribution of our system is checked by the concept of Herrera's cracking and the condition of causality is all around fulfilled for our models. The adiabatic index of our specific configuration is greater than $4 / 3$ in all interior points of the system and the mass-to-radius ratio in our situation is determined also lies within the Buchdahl limit i.e. $M / R \leq 4 / 3(\approx 0.444)$. We explore the physical characteristics based on the analytical model developed for relativistic compact stellar spheres inside the framework of the general theory of relativity. The evaluated mass and radius are in close concurrence with the observational information. We show that various physical characteristics of the known strange spherical object, viz. PSR J1614-2230, Vela X-1, 4U 1608-52, PSR J1903+327, 4 U 1820-30, Cen X-3, Her X-1, and SAX J1808.4-3658, can be described by the current model.
\end{abstract}

\footnotetext{
a e-mail: abdelghani.errehymy@gmail.com

b e-mail: m_daoud@hotmail.com

c e-mail: hassayout@yahoo.fr
}

\section{Introduction}

In the midst of the sphere's main sequence, hydrogen fuel combustion happens in the central zone in order to maintain the equilibrium of the star corresponding to the internal gravitational force. Burned hydrogen is the most productive wellspring of energy for any sphere assembled in principle and continues easily with no ferocious occasions, providing the star with the primary gauge of external pressure as well as the development of the helium center. However, beyond the combustion of Hydrogen, all other atomic combustion phases such as Helium combustion, Carbon combustion may happen on some cataclysmic occasions, for instance, planetary cloud, an explosion of supernovae joined by huge mass expulsion. In these occasions, the sphere center is too much packed prompting the configuration of relativistic compacts stellar spheres such as neutron star, white dwarfs, and black holes. Besides, the destiny of the packed center will be dictated by the underlying mass of its sphere of ancestor. The matter is to a great degree thick for the previously mentioned relativistic compacts stellar spheres in this way showing some extraordinary conduct and brings about unusual variation in space-time structure throughout it. The extraordinary states of energy density and pressure interior the sphere of the neutron can cause the neutron stage change into bosons, hyperons, and other strange quark matter. The more rigid states equation is necessary to clarify the conduct of the strange matter of the quark and other exotic states of matter [1-4]. The modeling of ultra-dense configurations in astrophysics of relativistic has aroused huge concern for astrophysicists in recent decades. The ongoing advances in observational procedures have uncovered numerous characteristics of the relativistic compacts stellar spheres yet at the same time; numerous lacunae are there to be settled. The hypothetical investigation of the nature and exact constitution of com- 
pact relativistic stellar spheres within the framework of the general relativity hypothesis is of fundamental importance for astrophysics. It encourages researchers to understand the interactions of particles with high density and to study their different stages of development as well as their internal characteristics. The compactification parameter of dead objects exits them from the condition of isotropic and matter demonstrates the anisotropic behavior which explains the difference in many of the characteristics of the compact objects.

In order to comprehend the inside configuration and transformative phases of relativistic compact stellar spheres, the anisotropic fluid appropriation plays a principal goal. As the relativistic compact stellar spheres have heavy centers and their densities surpass the density of nuclear, along these lines, pressure should be anisotropic interior the relativistic compact stellar spheres [5,6]. In the anisotropic distribution of matter, it is seen that pressure is parceled into radial and tangential constituents. In this case, numerous specialists have studied the features of dense relativistic compact stellar spheres with anisotropic fluid geometry. Hossein et al. [7] examined the highlights of anisotropic relativistic spheres with constant of cosmology and discovered stable astrophysical spheres configuration. Kalam et al. [8] analyzed the relativistic modeling of anisotropic neutron spheres and verified the availability of stability, anisotropy estimation and energy conditions with quintessence dark energy. Paul and Deb [9] detailed new exact solutions of relativistic compact stellar spheres for anisotropic matter distribution, which keep up the equilibrium of hydrostatic.

In relativistic compact stellar spheres, the more dense neutron spheres can additionally be fell to frame a black hole while for less dense neutron spheres, it is possible to transform into a quark sphere. It is predicted that the inside matter appropriation of quark spheres is expected to be processed using the state equation of the MIT quark bag model $[10,11]$. In recent years, the investigation of quark spheres has roused numerous physicists to examine its characteristics and in addition, their inside configuration. In this way, Bhar [12] analyzed features of PSR J1614-2230, 4U1820-30 and SAX J 1808.4-3658 spheres using the state equation of MIT quark bag model and gotten stability structure of these spheres. Rahaman et al. [13] suggested another mass insertion expression for the candidates of strange sphere with the state equation of MIT-Bag model and researched the physical conduct of spheres from $6 \mathrm{~km}$ to the limit surface. Different all around carried on solutions of Einstein's field equations have been investigated by forcing the barotropic state equation on the inside of the spherical object. Sharma and Ratanpal [14] have been proposed a relativistic astrophysical model conceding a quadratic state equation. Malaver $[15,16]$ examined a quadratic state equation for the distribution of matter and proposed special shapes for line element potentials in order to build compact spherical object mod- els. a few latest models of anisotropy for compact spherical objects with a distribution of strange matter incorporate the consequences of Sharma and Maharaj [17] and Lobo [18] with a barotropic state equation. Komathiraj and Maharaj [19] studied the anisotropic compact spherical object models of quark spheres obeying the state equation of the MIT bag model. However, can be noted that recent works concerning the distribution of the anisotropic matter, where a few specialists have considered the anisotropy is related to compact stellar objects [20-41].

Our objective in this paper is to present a new interior anisotropic solution to the Einstein field equations relativistic compact stellar sphere by using a linear state equation of the form $p_{r}=\alpha \rho-\beta$. We investigate the physical properties from the analytical model developed for compact spheres inside the framework of the general theory of relativity. The estimated mass and radius are in close agreement with the observational data. We show that various physical properties of the known strange spherical object, such as PSR J16142230, Vela X-1, 4U 1608-52, PSR J1903+327, 4U 1820-30, Cen X-3, Her X-1, and SAX J1808.4-3658, can be portrayed by our model.

The outline of this article is as follows: In Sect. 2, we present the interior metric and the corresponding Einstein field equations of anisotropic fluid distributions. The Generalized anisotropic solutions for compact spherical object are discussed in Sect. 3. The other characteristics are given in the Sects. 4 and 5, and a comparative study are given in Sect. 6. Concluding remarks close this paper.

\section{Interior solution and Einstein field equations of anisotropic fluid distributions}

We will consider a model which portrays a static spherically symmetric anisotropic fluid configuration governed by the linear equation of state of type $p=\alpha \rho-\beta$. The interior of static spherically symmetric objects is described by the canonical line element

$d s^{2}=-e^{\nu(r)} d t^{2}+\left(1+\frac{r^{2}}{R^{2}}\right) d r^{2}+r^{2} d \Omega^{2}$,

where $d \Omega^{2}=d \vartheta^{2}+\sin ^{2} \vartheta d \varphi^{2}$ is the metric on the unit 2-sphere, $v(r)$ is arbitrary functions of the radial parameter $r$ yet to be established. Also, having a momentum-energy tensor depicted for the astrophysical fluid filling the inside of the object has the standard form

$T_{\mu \nu}=\left(\rho+p_{t}\right) u_{\mu} u_{v}+p_{t} g_{\mu \nu}+\left(p_{r}-p_{t}\right) v_{\mu} v_{\nu}$,

where $\rho(r)$ is the energy density, $p_{r}$ is the radial pressure measured in the direction of $v_{\mu}, p_{t}$ is the transverse pressure measured in the orthogonal direction of $v_{\mu}, u_{\mu}$ is the vector 4 -velocity, $v_{\mu}$ is the space-like vector in the radial direction. 
Einstein's field equations for the line element (1), appropriately, are gotten as (we set $G=c=1$ )

$$
\begin{aligned}
\rho(r)= & \frac{1}{8 \pi R^{2}}\left(3+\frac{r^{2}}{R^{2}}\right)\left(1+\frac{r^{2}}{R^{2}}\right)^{-2}, \\
p_{r}(r)= & \frac{1}{8 \pi}\left\{\left(\frac{1}{r} \frac{d v(r)}{d r}+\frac{1}{r^{2}}\right)\left(1+\frac{r^{2}}{R^{2}}\right)^{-1}-\frac{1}{r^{2}}\right\}, \\
p_{t}(r)= & \frac{1}{8 \pi}\left\{\left(\frac{1}{2} \frac{d^{2} v(r)}{d r^{2}}+\frac{1}{2 r} \frac{d v(r)}{d r}\right.\right. \\
& \left.+\frac{1}{4}\left(\frac{d v(r))}{d r}\right)^{2}\right)\left(1+\frac{r^{2}}{R^{2}}\right)^{-1} \\
& \left.-\frac{1}{R}\left(1+\frac{r}{2} \frac{d v(r))}{d r}\right)\left(1+\frac{r^{2}}{R^{2}}\right)^{-2}\right\} .
\end{aligned}
$$

At this stage, we have a system of equations constituted by three equations, specifically, the field Eqs. (3)-(5), and four unknowns i.e., $\rho(r), p_{r}(r), p_{t}(r)$ and $v(r)$. By reasonably picking any of these unknown parameters, the system might be determined. Our goal here is to build up a model for compact object and, in this manner; it is helpful now to present the fluid with the most straightforward type of the compact object state equation in the specific form

$p_{r}=\alpha \rho-\beta$,

with $\alpha$ is real such that $0<\alpha \leq 1$, and $\beta=\alpha \rho_{s}$ is the surface density [17]. Replacing Eq. (6) in Eq. (4) and incorporating, we choose the undetermined metric expression $v(r)$ in the form

$$
\begin{aligned}
v(r)= & \alpha \ln \left(r^{2}+R^{2}\right) \\
& -\frac{2 \pi \beta}{R^{2}} r^{2}\left(r^{2}+2 R^{2}-\frac{1}{3 \pi \beta}\right)+v_{1},
\end{aligned}
$$

and $v_{1}$ is the integration constant.

From the above system Eqs. (3)-(5) and utilizing the Eq. (7), we verify that the physical parameters for this model are written as :

$$
\begin{aligned}
& \rho(r)=\frac{1}{8 \pi R^{2}}\left(3+\frac{r^{2}}{R^{2}}\right)\left(1+\frac{r^{2}}{R^{2}}\right)^{-2}, \\
& p_{r}(r)=\frac{1}{8 \pi R^{2}}\left\{\alpha\left(3+\frac{r^{2}}{R^{2}}\right)\left(1+\frac{r^{2}}{R^{2}}\right)^{-2}-8 \pi \beta R^{2}\right\},
\end{aligned}
$$

$$
\begin{aligned}
& p_{t}(r)=\frac{\left(1+\left(\frac{r}{R}\right)^{2}\right)^{-2}}{24 \pi R^{2}}\left\{\left(1+\left(\frac{r}{R}\right)^{2}\right)\right. \\
& \times\left[64 \pi^{2} R^{4} \beta^{2}\left(\frac{r}{R}\right)^{6}+\left(128 \pi^{2} R^{4} \beta^{2}-16 \pi R^{2} \beta\right.\right. \\
& \left.-8 \pi R^{2} \beta\left(1+\left(\frac{r}{R}\right)^{2}\right)^{-1}\right)\left(\frac{r}{R}\right)^{4}
\end{aligned}
$$

$$
\begin{aligned}
& +\left(64 \pi^{2} R^{4} \beta^{2}-16 \pi R^{2} \beta-8 \pi R^{2} \beta\left(1+\left(\frac{r}{R}\right)^{2}\right)^{-1}\right. \\
& \left.-3 \alpha\left(1+\left(\frac{r}{R}\right)^{2}\right)^{-2}+9\left(1+\left(\frac{r}{R}\right)^{2}\right)^{-1}+4 \alpha\right)\left(\frac{r}{R}\right)^{2} \\
& \left.+3 \alpha\left(1+\left(\frac{r}{R}\right)^{2}\right)^{-2}+3 \alpha\left(1+\left(\frac{r}{R}\right)^{2}\right)^{-1}-8 \pi R^{2} \beta\right] \\
& +\left[6 \alpha\left(\left(3+\left(\frac{r}{R}\right)^{2}\right)\right)+\left(3 \alpha\left(1+\left(\frac{r}{R}\right)^{2}\right)^{-1}\right.\right. \\
& \left.\left.\left.+12 \pi \beta R^{2}\left(\left(1+\left(\frac{r}{R}\right)^{2}\right)\right)\right)\left(\frac{r}{R}\right)^{2}\right]\right\}
\end{aligned}
$$

\section{Generalized anisotropic solution for compact spherical object}

We notice that the physical properties of the model rely upon the constants $R, \alpha$ and $\beta$. We have to put proper limits on these parameters aiming that the model can depict a reasonable compact spherical object. To this end, in view of different physical prerequisites, let us currently break down the conduct of the physical parameters.

We assume that $a$ is the radius of the compact spherical object. At that point, from Eq. (8), the surface and focal densities are respectively acquired as

$$
\begin{aligned}
& \rho(a)=\rho_{s}=\frac{1}{8 \pi R^{2}}\left(3+\left(\frac{a}{R}\right)^{2}\right)\left(1+\left(\frac{a}{R}\right)^{2}\right)^{-2}, \\
& \rho(0)=\rho_{c}=\frac{3}{8 \pi R^{2}} .
\end{aligned}
$$

We likewise have

$\rho^{\prime}(r)=-\frac{r}{4 \pi R^{4}}\left(5+\left(\frac{r}{R}\right)^{2}\right)\left(1+\left(\frac{r}{R}\right)^{2}\right)^{-3}<0$

$\rho^{\prime}(r=0)=0$

$\rho^{\prime \prime}(r=0)=-\frac{5}{4 \pi R^{4}}<0$

the prime signifies the differentiation on $r$. Clearly, the density is greatest at the core of the compact spherical object and it diminishes radially outward.

Similarly, the radial pressure can be written as

$$
\begin{aligned}
& p_{r}^{\prime}(r)=-\frac{\alpha r}{4 \pi R^{4}}\left(5+\left(\frac{r}{R}\right)^{2}\right)\left(1+\left(\frac{r}{R}\right)^{2}\right)^{-3}<0, \\
& p_{r}^{\prime}(r=0)=0 \\
& p_{r}^{\prime \prime}(r=0)=-\frac{5 \alpha}{4 \pi R^{4}}<0
\end{aligned}
$$

which indicates that the radial pressure additionally diminishes from the core of the compact spherical object towards the limit. In this manner, the radial pressure and the energy density are all around carried on in the inside of the astrophys- 


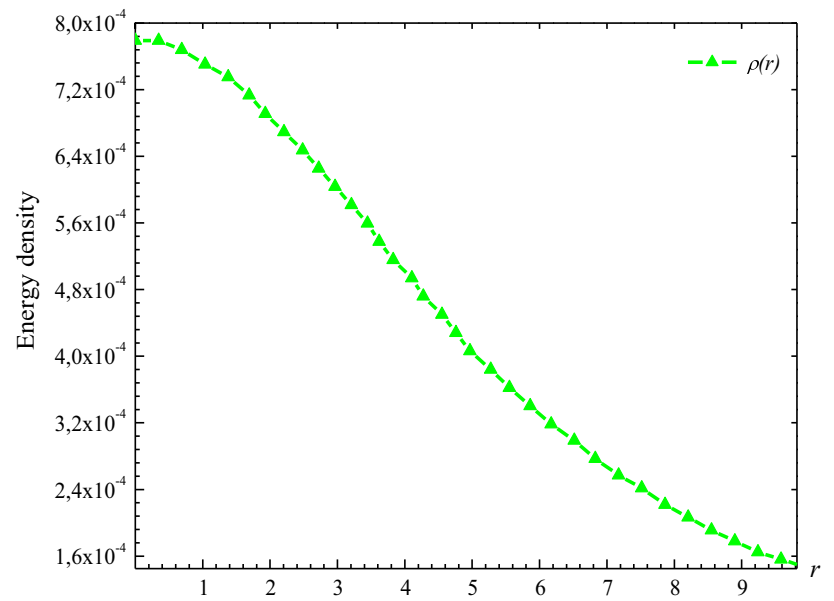

Fig. 1 Profile of the matter density $\rho$ with the radial coordinate $r$ of our model for spherical object Cen X-3

ical configuration. Behaviors of the energy density $\rho(r)$, the radial pressure $p_{r}(r)$ and the tangential pressure $p_{t}(r)$ have been presented in Figs. 1, 2 and 3, respectively. It is to be noted here that, like the slope in Maurya et al. $[32,39]$ which arguably described the SMC X-1, Herx X-1, 4U 1538-52 and RXJ 1856-37, and include anisotropy which vanishes at the boundary, the equation of state becomes much gentler, i.e. $d p_{r} / d \rho$ equals the constant parameter $\alpha$.

Using Eqs. (9) and (10) we get

$$
\begin{aligned}
& \Delta(r)=\frac{\left(1+\left(\frac{r}{R}\right)^{2}\right)^{-2}}{24 \pi R^{2}} \\
& \times\left\{( 1 + ( \frac { r } { R } ) ^ { 2 } ) \left[64 \pi^{2} R^{4} \beta^{2}\left(\frac{r}{R}\right)^{6}\right.\right. \\
& +\left(128 \pi^{2} R^{4} \beta^{2}-16 \pi R^{2} \beta\right. \\
& \left.-8 \pi R^{2} \beta\left(1+\left(\frac{r}{R}\right)^{2}\right)^{-1}\right)\left(\frac{r}{R}\right)^{4} \\
& +\left(64 \pi^{2} R^{4} \beta^{2}-16 \pi R^{2} \beta-8 \pi R^{2} \beta\left(1+\left(\frac{r}{R}\right)^{2}\right)^{-1}\right. \\
& \left.-3 \alpha\left(1+\left(\frac{r}{R}\right)^{2}\right)^{-2}+9\left(1+\left(\frac{r}{R}\right)^{2}\right)^{-1}+4 \alpha\right)^{2}\left(\frac{r}{R}\right)^{2} \\
& \left.+3 \alpha\left(1+\left(\frac{r}{R}\right)^{2}\right)^{-2}+3 \alpha\left(1+\left(\frac{r}{R}\right)^{2}\right)^{-1}-8 \pi R^{2} \beta\right] \\
& +\left[6 \alpha\left(\left(3+\left(\frac{r}{R}\right)^{2}\right)\right)+\left(3 \alpha\left(1+\left(\frac{r}{R}\right)^{2}\right)^{-1}\right.\right. \\
& \left.\left.\left.+12 \pi \beta R^{2} \times\left(\left(1+\left(\frac{r}{R}\right)^{2}\right)\right)\right)\left(\frac{r}{R}\right)^{2}\right]\right\} \\
& -\frac{1}{8 \pi R^{2}}\left\{\alpha\left(3+\frac{r^{2}}{R^{2}}\right)\left(1+\frac{r^{2}}{R^{2}}\right)^{-2}-8 \pi \beta R^{2}\right\} . \quad(19)
\end{aligned}
$$

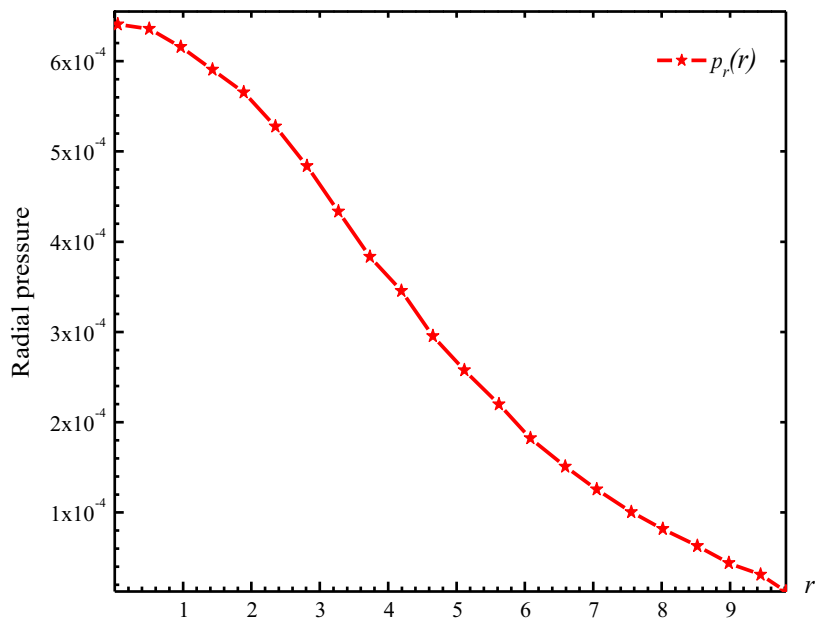

Fig. 2 Profile of the radial pressure $p_{r}$ with the radial coordinate $r$ of our model for spherical object Cen X-3

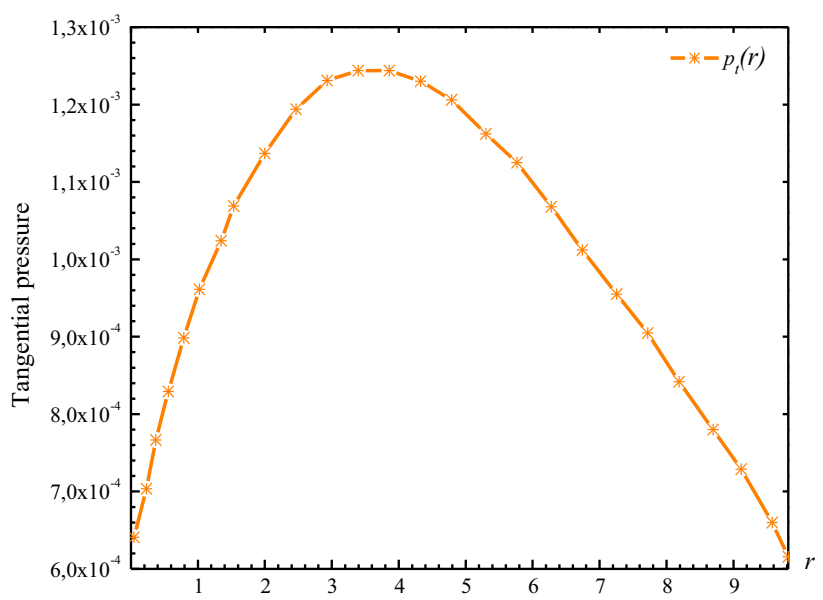

Fig. 3 Profile of the tangential pressure $p_{t}$ with the radial coordinate $r$ of our model for spherical object Cen X-3

$\Delta(r)=p_{t}(r)-p_{r}(r)$ is signified as the anisotropic parameter. It is a measure of the anisotropic pressure of the fluid including the compact spherical objet. $\Delta(r)=0$ matching to the special situation of a isotropic pressure spherical object. Notice that $2 \Delta(r) / r$ symbolizes a force appropriate to the anisotropic nature of the astrophysical form, which is repulsive, i.e., being outward directed if $\Delta(r)>0$, and attractive if $\Delta(r)<0$. We notice that our model is necessarily anisotropic for $\Delta(r) \neq 0$ in general, even in the simplest case of the distribution of matter. Some treatments of the physical properties of anisotropic objects in general relativity include investigations of Maharaj and Chaisi [42], Dev and Gleiser [43,44], Mak and Harko [45,46], and Chaisi and Maharaj $[47,48]$. Figure 4 illustrates the nature of the anisotropic pressure at the spherical object inside for a specific case. 


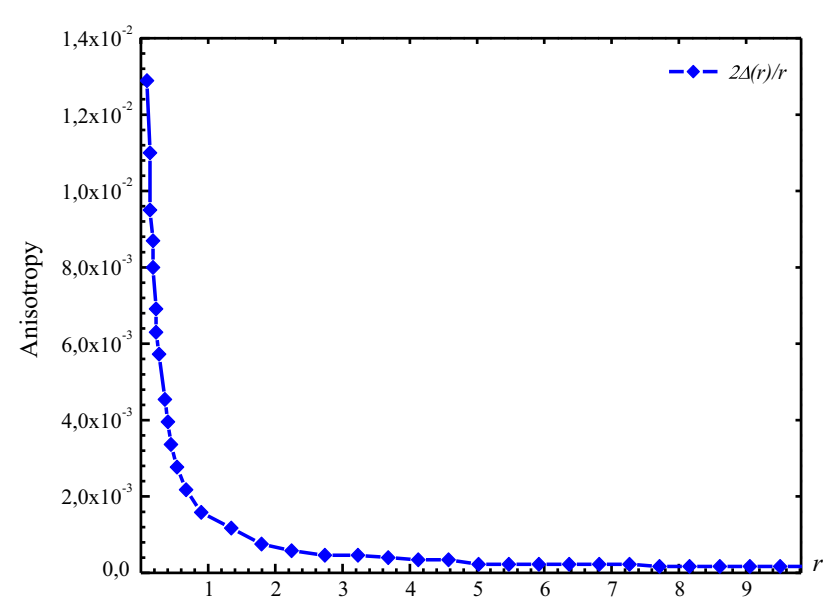

Fig. 4 Profile of the anisotropy parameter, $2 \Delta / r$, with the radial coordinate $r$ of our model for spherical object Cen X-3

The condition that the anisotropic parameter $\Delta(r)$ ought to vanish at the core $(r=0)$ yields

$3 \alpha-8 \pi R^{2} \beta=0$

which can be utilized to compute the constant $\beta$ for a specific choice of the constant $\alpha$.

\section{Matching conditions}

In this part, we compare the interior space-time $\left(\mathcal{M}_{-}\right)$ with the exterior space-time $\left(\mathcal{M}_{+}\right)$depicted by the external Schwarzschild solution in the approaching isotropic coordinates at the limit of the spherical object $r=a$, the inside metric ought to be matched to the Schwarzschild outside metric. The development of the metric functions over the limit surface yields

$$
\begin{aligned}
& \left(1+\left(\frac{a}{R}\right)^{2}\right)^{-1}=1-\frac{2 M(R)}{a}, \\
& v(r=a)=\ln \left(1-\frac{2 M(R)}{a}\right)=\alpha \ln \left(a^{2}+R^{2}\right) \\
& -2 \pi \beta\left(\frac{a}{R}\right)^{2}\left(a^{2}+2 R^{2}-\frac{1}{3 \pi \beta}\right),
\end{aligned}
$$

where $M(\mathrm{R})$ denotes the total mass of the spherical object of radius $\mathrm{R}$.

From Eq. (21), we get the expression for the compactness of the spherical object as

$$
\frac{M(R)}{a}=\frac{1}{2}\left(\frac{a}{R}\right)^{2}\left(1+\left(\frac{a}{R}\right)^{2}\right)^{-1} .
$$

We consider standard matter with a spherically symmetric anisotropic fluid where the radial pressure must be finite and positive interior the spherical object, and vanishes at the limit $r=a$ of the spherical object. This later condition that the radial pressure must vanish at the limit $\left(p_{r}(r=a)=0\right)$ gives

$\beta=\frac{\alpha}{8 \pi R^{2}}\left(3+\left(\frac{a}{R}\right)^{2}\right)\left(1+\left(\frac{a}{R}\right)^{2}\right)^{-2}$,

which imposes a limitation of the parameters $\alpha$ which can be solved in case we indicate the radius of the spherical object.

\section{Physical properties of the spherical objects}

In this section, we will discuss various physical characteristics of strange spherical objects using the previously proposed model.

\subsection{Stability of the system}

\subsubsection{The Tolman-Oppenheimer-Volkoff equation}

To examine the stability of the framework, we verified the stability equation given by Tolman [49], Oppenheimer and Volkoff [50]. The Tolman-Oppenheimer-Volkoff (TOV) equation represents the equilibrium condition of a spherical object subject to the gravitational force, anisotropy force and hydrostatic force. The generalized TOV equation can be composed as follows [20,51]:

$\frac{d p_{r}}{d r}+\frac{1}{2}\left(\rho+p_{r}\right) \frac{d v}{d r}+\frac{2}{r}\left(p_{r}-p_{t}\right)=0$.

We rewrite this Eq. (25) in the form

$$
\begin{gathered}
-\frac{M_{G}}{r^{2}}\left(\rho+p_{r}\right) \exp \left(\frac{\lambda-v}{2}\right) \\
-\frac{d p_{r}}{d r}+\frac{2}{r}\left(p_{t}-p_{r}\right)=0,
\end{gathered}
$$

where $M_{G}$ is the effective gravitational mass inside a spherical object of radius $r$ and is given by the Tolman-Whittaker procedure as follows

$M_{G}=\frac{1}{2} r^{2} \exp \left(\frac{v-\lambda}{2}\right) \frac{d v}{d r}$.

Finally the modified TOV equation in a simply form given by above Eq. (26), describes the equilibrium condition for anisotropic fluid spheres, the three forces are reactivated, namely, the gravitational $\left(F_{g}\right)$, the hydrostatics $\left(F_{h}\right)$ and the anisotropic $\left(F_{a}\right)$ forces are given by :

$F_{g}+F_{h}+F_{a}=0$,

where

$F_{g}=-\frac{1}{2}\left(\rho+p_{r}\right) \frac{d v}{d r}$,

$F_{h}=-\frac{d p_{r}}{d r}$, 


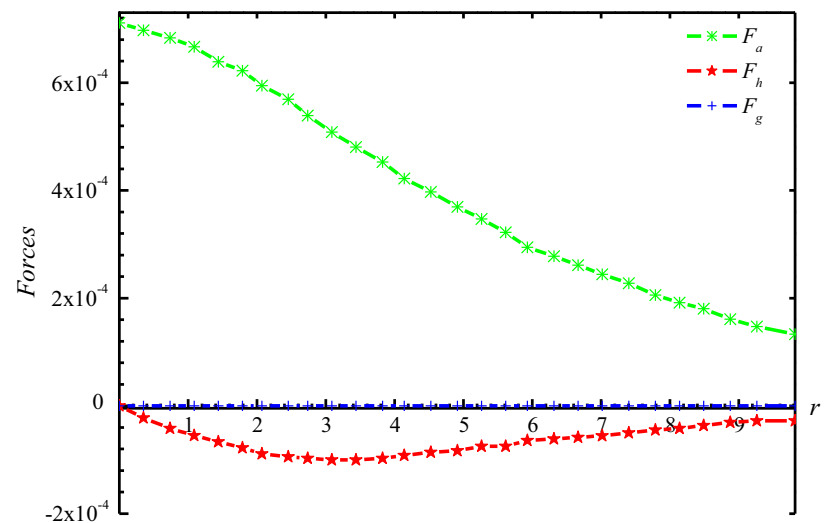

Fig. 5 Profile of the three different forces, $F_{a}, F_{h}$ and $F_{g}$ against the radial coordinate $r$ of our model for spherical object Cen X-3, with $M / a=0.33$

$F_{a}=\frac{2}{r}\left(p_{t}-p_{r}\right)$

To make simpler these Eqs. (29)-(31) alluded above, we have plotted the variations of $F_{g}, F_{h}$, and $F_{a}$ are the exhibit in Fig. 5. This figure shows that our proposed model is in static equilibrium is practicable for the reason that to pressure gravitational, hydrostatic and anisotropy forces.

\subsubsection{The status of the speed of sound within the system}

To check the potential stability or instability of our system we have used the concept of Herrera's cracking [52]. The causality condition determines the physical acceptability admitted for the fluid distribution which requires $0<v_{s r}^{2} \leq 1$ and $0<v_{s t}^{2} \leq 1$. According to the condition of Herrera [52] and Andréasson [53] for the stability of the distribution of matter, the region for which $\left|v_{s t}^{2}-v_{s r}^{2}\right| \leq 1$ i.e. no cracking is a potentially stable region. In Fig. 6, we observe that $\left|v_{s t}^{2}-v_{s r}^{2}\right| \leq 1$ in the region for which $r \leq 9.819$. This implies that our system meets all these conditions and that it is potentially stable in this region. The tangential and radial speeds of sound are defined respectively as $v_{s t}=\sqrt{d p_{t} / d \rho}$ and $v_{s r}=\sqrt{d p_{r} / d \rho}$.

\subsubsection{Adiabatic index}

According to Heintzmann and Hillebrandt [54], an anisotropic compact spherical object model will be stable if $\Gamma>4 / 3$ everywhere in the interior of the spherical object where the adiabatic index $\Gamma$ is defined as

$\Gamma=\left(\frac{\rho+p_{r}}{p_{r}}\right) \frac{d p_{r}}{d \rho}$.

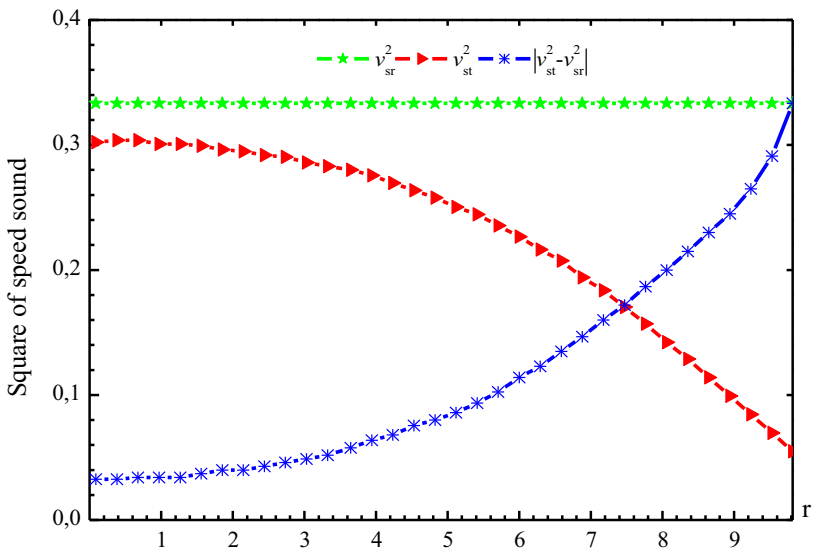

Fig. 6 Profil of the square of radial $v_{s r}^{2}$ and tangential $v_{s t}^{2}$ speed of sound and modulus of difference of the square of speed of sound $\left|v_{s t}^{2}-v_{s r}^{2}\right|$ with the radial coordinate $r$ of our model for spherical object Cen X-3, for $\beta=110.66 \mathrm{Mev} / \mathrm{fm}^{-3}$ and $\alpha=0.333$



Fig. 7 Profile of the adiabatic index, $\Gamma$, with the radial coordinate $r$ of our model for spherical object Cen X-3

We rewrite the above equation in the form

$\Gamma=2 \alpha \frac{\left(3+\left(\frac{r}{R}\right)^{2}\right)-4 \pi \beta R^{2}\left(1+\left(\frac{r}{R}\right)^{2}\right)^{2}}{\left(3+\left(\frac{r}{R}\right)^{2}\right)-8 \pi \beta R^{2}\left(1+\left(\frac{r}{R}\right)^{2}\right)^{2}}$

The adiabatic index, $\Gamma$, with the radial coordinate $r$ of our model for spherical object Cen X-3 is motivated by the fact that it gives a monotonically increasing function in the stellar inside. Similar forms for the adiabatic index have earlier been considered by Maurya et al. [32] for anisotropic fluid stars. Figure 7 clearly shows that the adiabatic index of our specific configuration is greater than $4 / 3$ in all interior points of the specific configuration. This characteristic obviously indicates that the system in this work is stable. 
Table 1 Physical parameters for different anisotropic astrophysical models for $\alpha=0.333$

\begin{tabular}{|c|c|c|c|c|c|c|c|}
\hline Observed objects & $\begin{array}{l}\text { Radius } \\
a(\mathrm{~km})\end{array}$ & $\begin{array}{l}M \\
\left(M_{\odot}\right)\end{array}$ & $\begin{array}{l}\text { Radius } \\
R(\mathrm{~km})\end{array}$ & $M / a$ & $\begin{array}{l}\beta \\
\left(\mathrm{MeV} \mathrm{fm}^{-3}\right)\end{array}$ & $\begin{array}{l}\rho(0) \\
\left(\begin{array}{l}\times 10^{15} \\
\mathrm{gm} \mathrm{cm}^{-3}\end{array}\right)\end{array}$ & $\begin{array}{l}p_{r}(0) \\
\left(\begin{array}{l}\times 10^{35} \\
\text { dyne } \mathrm{cm}^{-3}\end{array}\right.\end{array}$ \\
\hline PSR J1614-2230 & 10.300 & 1.970 & 9.69 & 0.280 & 106.66 & 1.421 & 2.482 \\
\hline Vela X-1 & 10.143 & 1.770 & 9.56 & 0.260 & 108.00 & 1.299 & 2.116 \\
\hline $4 \mathrm{U} 1608-52$ & 10.105 & 1.740 & 9.52 & 0.255 & 110.60 & 1.272 & 2.035 \\
\hline PSR J1903+327 & 10.017 & 1.667 & 9.43 & 0.245 & 112.07 & 1.198 & 1.813 \\
\hline $4 \mathrm{U} 1820-30$ & 10.000 & 1.580 & 9.31 & 0.235 & 114.90 & 1.113 & 1.558 \\
\hline Cen X-3 & 9.819 & 1.490 & 9.17 & 0.225 & 118.26 & 1.042 & 1.347 \\
\hline Her X-1 & 6.700 & 0.880 & 8.10 & 0.168 & 132.96 & 1.381 & 4.145 \\
\hline SAX J1808.4-3658 & 7.070 & 1.435 & 7.95 & 0.299 & 217.08 & 4.808 & 14.425 \\
\hline
\end{tabular}

\subsection{Energy conditions}

An acceptable compact spherical object must satisfy the energy conditions, in specific, the strong (SEC), weak (WEC), null (NEC) and dominant (DEC) energy conditions defined below:

SEC: $\rho(r)+p_{r}(r)+2 p_{t}(r) \geq 0$,

WEC: $\rho(r)+p_{r}(r) \geq 0$,

NEC: $\rho(r)+p_{t}(r) \geq 0$,

DEC: $\rho(r) \geq\left|p_{r}(r)\right|,\left|p_{t}(r)\right|$.

For the specific astrophysical configuration generated here, the availability of inequalities (42)-(45) has been highlighted some limitations on the model parameters. In our model, applying these energy conditions to the center $(r=0)$ we obtain the following limits:

SEC: $\rho(0)+p_{r}(0)+2 p_{t}(0) \geq 0, \Rightarrow \beta \leq \frac{9+57 \alpha}{40 \pi R^{2}}$,

i.e. $\beta \leq\left(\frac{3+19 \alpha}{5}\right) \rho_{c}$,

WEC: $\rho(0)+p_{r}(0) \geq 0, \Rightarrow \quad \beta \leq \frac{3(\alpha+1)}{8 \pi R^{2}}$,

i.e. $\beta \leq(\alpha+1) \rho_{c}$,

NEC: $\rho(0)+p_{t}(0) \geq 0, \Rightarrow \quad \beta \leq \frac{3(8 \alpha+3)}{8 \pi R^{2}}$,

i.e. $\beta \leq(8 \alpha+3) \rho_{c}$,

DEC: $\rho(0) \geq\left|p_{r}(0)\right|,\left|p_{t}(0)\right|$, i.e. $\beta \leq(\alpha+1) \rho_{c}$,

or $\beta \leq(8 \alpha+3) \rho_{c}$,

where $\rho(0)=\rho_{c}$ is the central density. We have demonstrated the profiles of all the previously mentioned energy inequalities and remarkably our system is predictable with all the energy conditions. The values of the model parameters for various astrophysical configurations agree with these limits, as on view in Table 1.

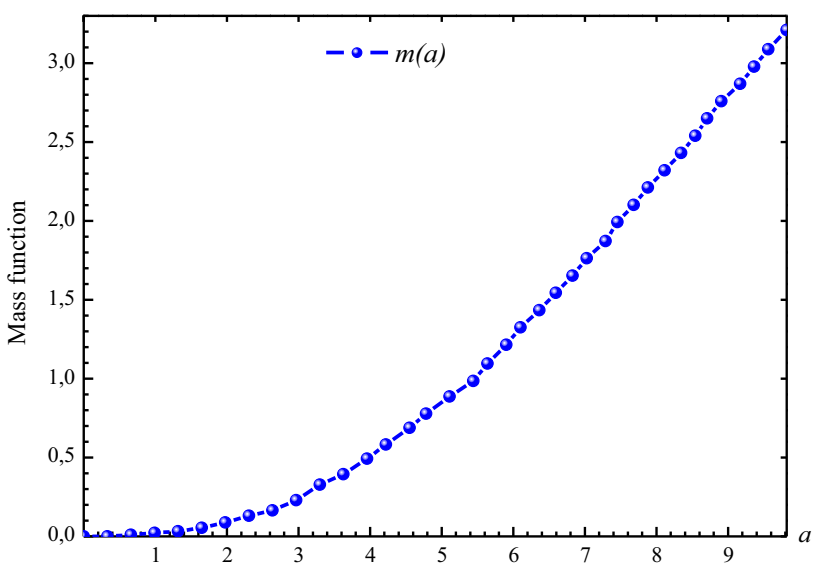

Fig. 8 Behaviour of the mass relation with the radial coordinate $a$ of our model for spherical object Cen X-3

\subsection{Mass function}

In our model, the mass function within the radius $a$ is obtained as

$m(a)=\int_{0}^{a} \rho(r) d r=\frac{a}{2}\left(\frac{a}{R}\right)^{2}\left(1+\left(\frac{a}{R}\right)^{2}\right)^{-1}$.

Since at $\lim _{a \rightarrow 0} m(a)=0$, recommend that the mass function is regular at the focal point of the star. The variation of the mass function is illustrated in Fig. 8, which demonstrates that the mass function is a positive and monotonically expanding physical quantity with respect to the radial coordinates $a$.

\subsection{Mass-radius ratio}

The compactification parameter of a spherically symmetric body is defined as the mass-radius function of the system, i.e. $u(a)=m(a) / a$. According to the condition of Buchdahl [55] for a $(3+1)$-dimensional perfect fluid spherically symmetric body, the maximum allowed mass-radius function is $M / R<$ $4 / 9$ where $R>2 M$. To see the best extent of the mass- 


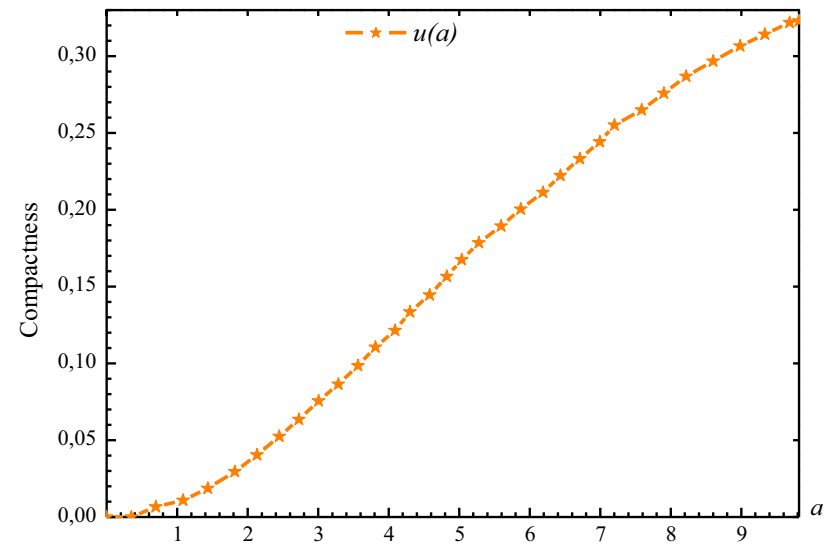

Fig. 9 Behaviour of the compactness relation with the radial coordinate $a$ of our model for spherical object Cen X-3

radius ratio for our model we best extent the compactification parameter of the system given by

$u(a)=\frac{1}{2}\left(\frac{a}{R}\right)^{2}\left(1+\left(\frac{a}{R}\right)^{2}\right)^{-1}$,

The variation of the compactness function has been shown in Fig. 9. This figure shows that the compactness function is a monotonic increasing function with respect to the radial coordinates $a$. We remark that the requirement on the greatest permitted mass-to-radius ratio in our situation is determined as $M / R=0.323$, which is like the isotropic fluid spherical object, i.e., $M / R \leq 4 / 9(\approx 0.444)$.

\subsection{Surface redshift function}

We have likewise specified the surface redshift function $\mathrm{z}_{\mathrm{s}}$ of a spherically symmetric body can be defined as

$z_{s}=\frac{1}{\sqrt{1-2 u(a)}}-1$,

which for the above studied model is given by

$z_{s}=\left(1+\left(\frac{a}{R}\right)^{2}\right)^{\frac{1}{2}}-1$.

Variation of the surface redshift function $z_{s}$ against to the radial coordinates $a$, as shown in Fig. 10. The greatest value permitted surface redshift $z_{s}$ in our case is 0.317 . The figure in like manner shows that the surface redshift function is limited within the stellar configuration.

\section{A comparative study}

To examine the physical characteristics based on the analytical model developed so far, we choose the spherical object



Fig. 10 Behaviour of the surface redshift expression with the radial coordinate $a$ of our model for spherical object Cen X-3

Cen X-3 as a delegate of the strange spherical objects possessing the following parameters: mass $m(a)=1.49 M_{\odot}$ and radius $a=9.819 \mathrm{~km}$ for $\beta=118.26 \mathrm{MeV} \mathrm{fm}^{-3}$ and $\alpha=0.333$.

Using the chosen mass and radius values shown in Table 1, we have shown various physical characteristics of the proposed configuration of strange compact objects. The observed masses shown in Table 1 are available in the following references [56-60]. We have highlighted the values of the constant $\beta$, especially for astrophysical configurations of strange spherical object candidates such as PSR J16142230, Vela X-1, 4U 1608-52, PSR J1903+327, 4U 1820-30, Her X-1, and SAX J1808.4-3658. We find that when the values of $\beta$ increase with $\alpha$ fixed at 0.333 , the astrophysical system becomes more compact and the density in the spherical object increases progressively with the increasing values of $\beta$. The observed value of the mass of Cen X-3 [56] is obtained for the astrophysical system to contract, that is to say the progressively decreasing radius values. However, from the point of view of a mathematically steady model, it gives the idea that an extensive variety of estimation of the constant $\beta$ is conceivable, which is coherent with the data of Relativistic Heavy Ion Collider (RHIC) and Super Proton Synchrotron (SPS). The obtained solutions are free of any singularity and exhibit a well-behaved nature within the generalized anisotropic solution for compact spherical object. This agrees with the results reported by Maurya et al. $[32,39,61]$. We have demonstrated that the anisotropy effects lead a wide range of results in the geometry and composition of an object. For instance, this may either yield a gentler equation of state or an object with various mass and radius. Our examination depends basically on the choice of a specific metric function such one given by the Eq. (7) yielding a linear equation of state. 


\section{Concluding remarks}

We have proposed a spherically symmetric model of anisotropic fluid for strange quark spheres which satisfies all conditions of physical reality and is devoid of focal singularity with the outer space-time being the Schwarzschild metric. We introduced this formalism to determine exact estimations of the radius of the different candidates of the strange sphere by using their observational mass and constant $\beta$ gave in Table 1 . We also discussed the stability analysis which verified that our model was completely satisfied with the conditions of causality and the concept of Herrera cracking. In a similar idea, in order to analyze the meaning of our model in the search for compact objects, we have considered various compact objects, including PSR J1614-2230, Vela X-1, 4U 1608-52, PSR J1903+327, 4U 1820-30, Her X-1, and SAX J1808.4-3658 and showed that, for the estimated radius, the masses of the object dictated by our model are close to the observed observation (see Refs. [56-60]). This generally suggests that the solution proposed in this work can be used as a feasible model for describing ultra-dense compact strange spheres.

Data Availibility Statement This manuscript has no associated data or the data will not be deposited. [Authors comment: This is a theoretical paper and this manuscript has non associated data. All the required theoretical data and the figures are already provided by the author.]

Open Access This article is distributed under the terms of the Creative Commons Attribution 4.0 International License (http://creativecomm ons.org/licenses/by/4.0/), which permits unrestricted use, distribution, and reproduction in any medium, provided you give appropriate credit to the original author(s) and the source, provide a link to the Creative Commons license, and indicate if changes were made. Funded by SCOAP ${ }^{3}$.

\section{References}

1. M. Alford et al., Astrophys. J. 629, 969 (2005)

2. B.V. Ivanov, Eur. Phys. J. C 77, 738 (2017)

3. A. Drago et al., Phys. Rev. D 89, 043014 (2014)

4. B.V. Ivanov, Eur. Phys. J. C 78, 332 (2018)

5. M. Ruderman, Annu. Rev. Astron. Astrophys. 10, 27 (1972)

6. B.V. Ivanov, Phys. Rev. D 65, 104011 (2002)

7. S.K.M. Hossein et al., Int. J. Mod. Phys. D 21, 1250088 (2012)

8. M. Kalam et al., Astrophys. Sp. Sci. 349, 865 (2014)
9. B.C. Paul, R. Deb, Astrophys. Sp. Sci. 354, 421 (2014)

10. E. Witten, Phys. Rev. D 30, 272 (1984)

11. K.S. Cheng et al., Int. J. Mod. Phys. D 7, 139 (1998)

12. P. Bhar, Astrophys. Sp. Sci. 357, 46 (2015)

13. F. Rahaman et al., Eur. Phys. J. C 74, 3126 (2014)

14. R. Sharma, B.S. Ratanpal. arXiv:1307.1439v1 (2013)

15. M. Malaver, Front. Math. Appl. 1, 9 (2014)

16. M. Malaver, Int. J. Mod. Phys. Appl. 2, 1 (2015)

17. R. Sharma, S.D. Maharaj, Mon. Not. R. Astron. Soc. 375, 1265 (2007)

18. F.S.N. Lobo, Class. Quantum Gravity 23, 1525 (2006)

19. K. Komathiraj, S.D. Maharaj, Int. J. Mod. Phys. D 16, 1803 (2007)

20. V. Varela et al., Phys. Rev. D 82, 044052 (2010)

21. F. Rahaman et al., Phys. Rev. D 82, 104055 (2010)

22. P. Fuloria, Eur. Phys. J. A 54, 179 (2018)

23. A. Errehymy, M. Daoud, Found. Phys. 1, 1-32, (2019)

24. M. Kalam et al., Eur. Phys. J. C 73, 2409 (2013)

25. S.K. Maurya, F. Tello-Ortiz, Eur. Phys. J. C 79, 85 (2019)

26. E. Morales, Tello-Ortiz, Eur. Phys. J. C 78, 841 (2018)

27. S.K. Maurya, Y.K. Gupta, S. Ray, Eur. Phys. J. C 77, 360 (2017)

28. A. Errehymy, M. Daoud, Mod. Phys. Lett. A 34, 1950030, (2019)

29. S.K. Maurya, S.D. Maharaj, Eur. Phys. J. C 77, 328 (2017)

30. E. Morales, F. Tello-Ortiz, Eur. Phys. J. C 78, 618 (2018)

31. S.K. Maurya, M. Govender, Eur. Phys. J. C 77, 420 (2017)

32. S.K. Maurya et al., Eur. Phys. J. C 76, 266 (2016)

33. G. E. Delgado et al. arXiv preprint arXiv:1807.10360 (2018)

34. F. Rahaman et al., Class. Quantum Gravity 28, 155021 (2011)

35. Bhar et al., Eur. Phys. J. C 75, 190 (2015)

36. F. Rahaman et al., Gen. Relativ. Gravity 44, 107 (2012)

37. M. Kalam et al., Eur. Phys. J. C 72, 2248 (2012)

38. P. Bhar et al., arXiv preprint arXiv:1605.01274 (2016)

39. S.K. Maurya et al., Astrophys. Sp. Sci. 363, 208 (2018)

40. P. Bhar et al., Eur. Phys. J. A 52, 312 (2016)

41. A. Errehymy et al., Eur. Phys. J. Plus 132, 497 (2017)

42. S.D. Maharaj, M. Chaisi, Gen. Relativ. Gravity 38, 1723 (2006)

43. K. Dev, M. Gleiser, Gen. Relativ. Gravity 34, 1793 (2002)

44. K. Dev, M. Gleiser, Gen. Relativ. Gravity 35, 1435 (2003)

45. M.K. Mak, T. Harko, Chin. J. Astron. Astrophys. 2, 248 (2002)

46. M.K. Mak, T. Harko, Proc. Roy. Soc. Lond. A 459, 393 (2003)

47. M. Chaisi, S.D. Maharaj, Gen. Relativ. Gravit. 37, 1177 (2005)

48. M. Chaisi, S.D. Maharaj, Pramana - J. Phys. 66, 609 (2006)

49. R.C. Tolman, Phys. Rev. 55, 364-373 (1939)

50. J.R. Oppenheimer, G. Volkoff, Phys. Rev. 55, 374-381 (1939)

51. J. Ponce de Léon, Gen. Relativ. Gravity 25, 25 (1993)

52. L. Herrera, Phys. Lett. A 165, 206 (1992)

53. H. Andréasson, Commun. Math. Phys. 288, 715 (2009)

54. H. Heintzmann, W. Hillebrandt, Astron. Astrophys. 38, 51 (1975)

55. H.A. Buchdahl, Phys. Rev. D 116, 1027 (1959)

56. M.L. Rawls et al., Astrophys. J. 730, 25 (2011)

57. T. Guver et al., ApJ 712, 964 (2010)

58. P.C.C. Freire et al., Mon. Not. R. Astron. Soc. 412, 2763 (2011)

59. T. Guver et al., ApJ 719, 1807 (2010)

60. P.B. Demorest et al., Nature 467, 1081 (2010)

61. S.K. Maurya et al., Phys. Rev. D 97, 044022 (2018) 